\title{
Flow-Injection-Based Miniaturized Quartz Crystal Microbalance
}

\author{
Jinxing Liang*, Jia Huang, Sheng Ding and Toshitsugu Ueda ${ }^{1}$ \\ Key Laboratory of Micro-Inertial Instrument and Advanced Navigation Technology, \\ Ministry of Education, School of Instrument Science and Engineering, Southeast University, \\ 2 Sipailou, Nanjing 210096, China \\ ${ }^{1}$ Graduate School of Information, Production and Systems, Waseda University, \\ 2-7 Hibikino, Wakamatsu-ku, Kitakyushu 808-0135, Japan
}

(Received June 6, 2013; accepted June 17, 2013)

Key words: flow injection, quartz crystal microbalance, high fundamental frequency, wet etching, miniaturization

In this research, we present the design and realization of a flow-injection-based highfundamental-frequency (HFF) quartz crystal microbalance (QCM) sensor. A well-known fact is that increasing the fundamental frequency of thickness-shear-mode (TSM) QCM would result in an increase in mass sensitivity when it is used as chemical sensors or biosensors. HFF QCM is designed and fabricated on a small $4 \times 4 \mathrm{~mm}^{2}$ AT-cut quartz crystal chip using an inexpensive wet etching process with an inverted-mesa structure. For actual applications in liquid, a flow-injection-based QCM system is established, which is composed of a syringe pump, a sample injector, and a custom-made microflow cell. HFF QCMs are achieved by decreasing the central area thickness of a quartz wafer, which is inversely proportional to the vibration frequency. The vibration characteristics of fabricated QCM chips, including resonance frequency, quality factor ( $\mathrm{Q}$ value), and equivalent circuit parameters, are measured using an impedance analyzer 4294A. HFF QCMs with high Q values of up to 29000 for $52 \mathrm{MHz}$ in air were achieved. Even if assembled into the custom-made microflow cell, QCMs show a $Q$ value beyond 23000 as measured using an impedance analyzer. These results demonstrate the successful design and fabrication of the flow cell, which does not degrade the performance of the QCM resonator. A Q value as high as 1000 of HFF QCM was observed even when pure water is flowed through the chip surface, which clearly demonstrated that the fabricated QCM and flow cell system could be used in liquid.

${ }^{*}$ Corresponding author: e-mail: j-liang@seu.edu.cn 


\section{Introduction}

The quartz crystal microbalance (QCM) is a well-known mass-sensitive tool, which is widely used as chemical sensors or biosensors owing to its high-temperature frequency stability and mass sensitivity. Principally, the QCM is a kind of resonator working in thickness-shear-mode (TSM) vibration, the fundamental frequency of which is inversely proportional to the wafer thickness. A mass change on the surface of QCM corresponds to a thickness change of the quartz substrate, resulting in a frequency shift. The frequency shift of QCM induced by the mass change is proportional to the square of the fundamental frequency, according to the following well-known Sauerbery equation: ${ }^{(1)}$

$$
\Delta f=-\frac{2 f_{0}^{2} \Delta m}{A \sqrt{\rho \mu}}
$$

where $\Delta f$ and $\Delta m$ are the frequency shift and mass change, respectively; $f_{0}$ is the fundamental frequency, $A$ is the excitation electrode area, and $\rho$ and $\mu$ are the density and shear modulus of the AT-cut quartz crystal, respectively. Accordingly, the mass sensitivity of QCM could be increased by increasing the fundamental frequency. Usually, HFF QCM utilizes an inverted-mesa structure, in which the central region is thinned for obtaining HFF vibration, and the outer ring retains its original thickness for maintaining the necessary mechanical strength needed for chip assembly and handling. Lin et al. firstly reported an ultrasensitive QCM working at $30 \mathrm{MHz}$ by chemically milling an AT-cut quartz crystal.(2) After that, many studies have been reported on the fabrication of the HFF QCM chip by wet etching ${ }^{(3,4)}$ and deep reactive ion etching (DRIE), ${ }^{(5,6)}$ flow-injection-based QCM system, ${ }^{(7-9)}$ and sensor applications. ${ }^{(9-13)}$ The miniaturization of the measurement system and that of the chip array are the development trends of HFF QCM. For the preparation of the QCM chip, wet etching is apparently simple and cheap; however, it is considered inappropriate for its further miniaturization owing to the complex anisotropic etched side wall profile of the AT-quartz wafer. ${ }^{(6)}$ DRIE has recently been reported and successfully demonstrated to fabricate a miniaturized QCM chip and array with a vertical side wall; however, this process is expensive and needs special etching equipment. The design of the flow cell is another important issue for the miniaturization of the QCM system, which should not affect the vibration characteristics of the QCM chip. A miniaturized flow cell, which is easy to assemble and change the QCM chip, is desired.

In this research, we present a novel QCM system, which is composed of a syringe pump, a sample injector and a custom-made microflow cell. Figure 1 shows the schematic of the proposed HFF QCM measurement system. The miniaturized HFF QCM chip is designed to have a square shape and fabricated by wet etching. The key feature of the flow cell is that excitation electrodes on the two side surfaces of QCM are led out by the pin contacting method, which makes it easy to change and modify the QCM chip. 


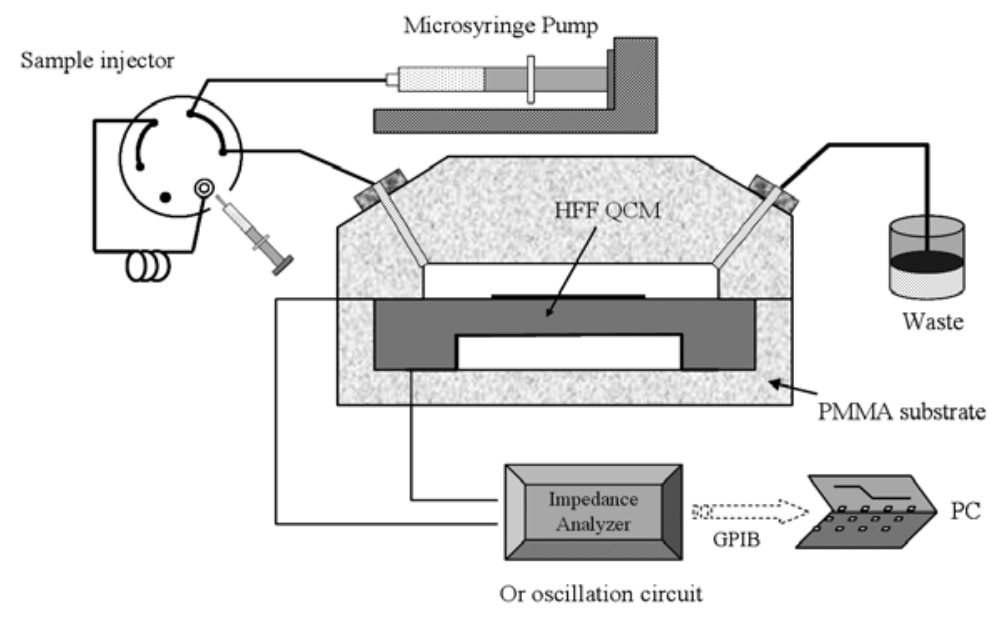

Fig. 1. Schematic of proposed flow-injection-based HFF QCM system.

\section{Experimental Methods}

\subsection{Design and fabrication of miniaturized QCM chip}

The dimensions of the QCM chip are designed to be $4 \times 4 \times 0.1 \mathrm{~mm}^{3}$, and the central vibration region is set to be $1.5 \mathrm{~mm}$ in diameter. The size of the excitation electrode is an important parameter related to the vibration characteristics of the QCM. The ratio of excitation electrode size to thickness of vibration area should be sufficiently large to ensure stable vibration; however, a very large electrode (namely, a small distance between the edges of the electrode and vibration area) would cause spurious vibrations. In this research, aiming to achieve 50-MHz-level QCM, the excitation electrode size is designed to be $0.5 \mathrm{~mm}$ in diameter. The fabrication process starts from a $100-\mu \mathrm{m}$-thick AT-cut wafer, and the detailed process flow is as follows: 1) washing quartz wafer using piranha solution $\left(\mathrm{H}_{2} \mathrm{SO}_{4}: \mathrm{H}_{2} \mathrm{O}_{2}=3: 1\right)$ at $110{ }^{\circ} \mathrm{C}$ for $\left.15 \mathrm{~min} ; 2\right)$ sputtering $\mathrm{Au} / \mathrm{Cr}$ metal films on the two sides of the wafer; 3 ) coating and patterning of photoresist to define the etching area (vibration area) on one side of the wafer, followed by etching $\mathrm{Au} / \mathrm{Cr}$ metal films; 4) coating and patterning of photoresist to define the excitation electrode; 5) etching quartz using saturated ammonium bifluoride solution at $\left.86^{\circ} \mathrm{C} ; 6\right)$ etching $\mathrm{Au} / \mathrm{Cr}$, followed by photoresist removal; and 7) sputtering $\mathrm{Au} / \mathrm{Cr}$ metal films on the etched side surface.

\subsection{Preparation of flow cell}

Figure 2 shows a detailed structure of the flow cell, which is composed of a cover, a substrate, and an intermediate layer of silicon rubber. Polymethyl methacrylate (PMMA) is selected as the cover and substrate material because it is light, transparent, and easy 

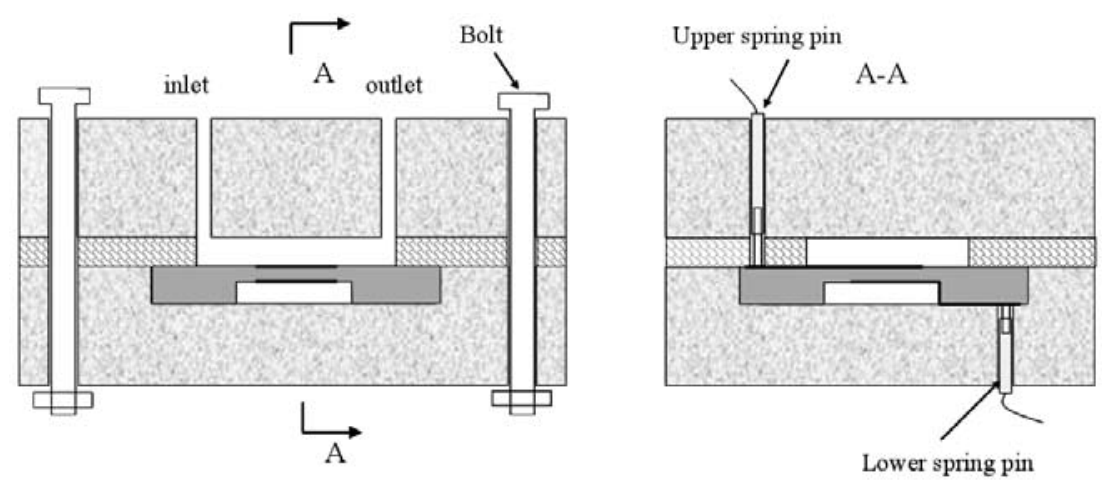

Fig. 2. Schematic of the designed flow cell.

to manufacture. The cover and substrate are fixed using two bolts. Three small through holes are opened in the cover part, one for the spring pin that is used to electrically lead one face electrode of the QCM chip, and other two holes as liquid solution inlet and outlet. A rectangular cavity is drilled in the substrate part, which is used to mechanically keep the QCM chip. One through hole is opened below the cavity to stabilize the spring pin, which is used to electrically lead the other face electrode of the QCM chip. The silicon rubber works not only for sealing the QCM chip, but also as a liquid solution container by punching a hole. The hole is larger than the excitation electrode and smaller than the QCM chip.

\subsection{Evaluation method}

An impedance analyzer 4294A is used to characterize the QCM chip and flow cell performance by measuring the frequency admittance value. Before being assembled into the flow cell, the vibration characteristics of the QCM chip, including the resonance frequency, $\mathrm{Q}$ value, and equivalent circuit parameters, are measured with the aid of a $16034 \mathrm{G}$ test fixture, as shown in Fig. 3. Under this condition, the measured result is considered to be the natural characteristic of the QCM resonator. After that, the QCM chip is assembled into the custom-made flow cell. The etched side is arranged facing the substrate and the unetched side is arranged facing the cover. This means that the thinned vibration area is kept free. The assembled QCM is electrically connected to the impedance analyzer via a $16047 \mathrm{E}$ adapter. To evaluate the quality of the flow cell, the QCM vibration characteristics are measured in air and compared with the previous measured result using 16034G. To investigate the mechanical and electrical behaviors of QCM in liquid media, pure water is flowed through the QCM surface using a syringe pump. Furthermore, to also confirm the function of the flow injection, $0.1 \mathrm{M}$ phosphatebuffered saline (PBS, PH 7.4) solution is introduced using the sample injector. 


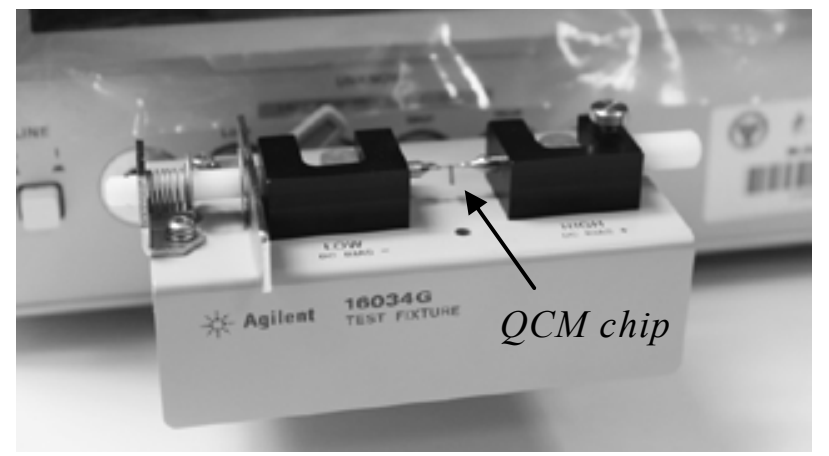

Fig. 3. Measurement setup using impedance analyzer with $16034 \mathrm{G}$ test fixture.

\section{Results and Discussion}

Figure 4 shows a typical example of the fabricated QCM chip. Figure 4(a) shows the upper-surface image, which is not etched and exposed to a liquid medium. The central Au metal film serves not only as the excitation electrode, but also as the interface for immobilizing biosensors or chemical sensors modifying films, such as antibodies and SAMs. Figure 4(b) shows the back-surface image, the center of which is etched to the desired thickness. For easy fabrication, the back side is fully covered by $\mathrm{Au} / \mathrm{Cr}$ metal films as the excitation electrode. Figure 4(c) shows a close-up SEM image of the etched area. Figure 5 shows the fabricated flow cell, which can be easily assembled. Furthermore, instead of conventional fixed soldering electrical connection or wire bonding connection, the QCM excitation electrodes are led out by placing the two spring pins in contact, which induces the flow cell reversible change of the QCM chip. Figure 6 shows the measured conductance near the fundamental resonance using the impedance analyzer 4294A. Figure 6(a) shows the result of the QCM chip fabricated in air using a test fixture, $16034 \mathrm{G}$. The fundamental resonance frequency is about $52 \mathrm{MHz}$, which is ten times higher than that of the traditional QCM. Generally, the $\mathrm{Q}$ value, $C_{0} / C_{1}$ ratio, and motional resistance are the main parameters for estimating the quality of the resonator. $^{(3)}$ The Q value of the fabricated $52 \mathrm{MHz}$ QCM chip, which is calculated by dividing the peak frequency by the frequency bandwidth at half the peak conductance, is 29000 in air, although the chip size and etched area are as small as $4 \times 4 \mathrm{~mm}^{2}$ and 1.5 $\mathrm{mm}$ in diameter, respectively. Particularly when considering that the $\mathrm{Q}$ value is inversely proportional to the fundamental frequency, this $\mathrm{Q}$ value is considerably high. The $C_{0} / C_{1}$ ratio is 398 , and the motional resistance is about $60 \Omega$. The detailed design, fabrication method, and evaluation results regarding higher frequency QCMs and the dependence of vibration characteristics on the excitation electrode size can be found in our previous conference report. ${ }^{(14)}$ Similar results have been reported in previous publications; ${ }^{(3,15)}$ however, this research has the advantages of small size over that shown in ref. 3, and 


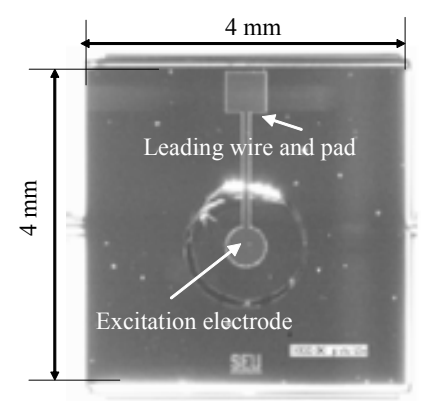

(a)

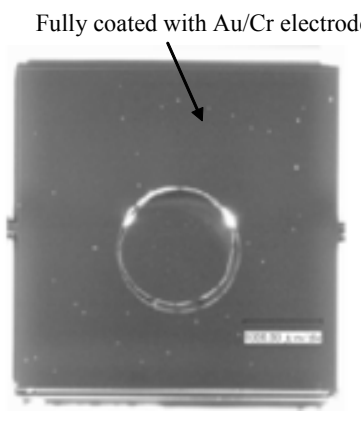

(b)

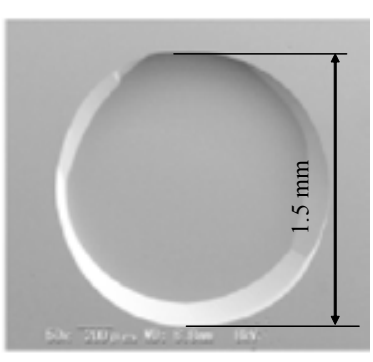

(c)

Fig. 4. Typical example images of fabricated HFF QCM chip: (a) upper side image, (b) back (etched) side image, and (c) close-up SEM image of etched area.

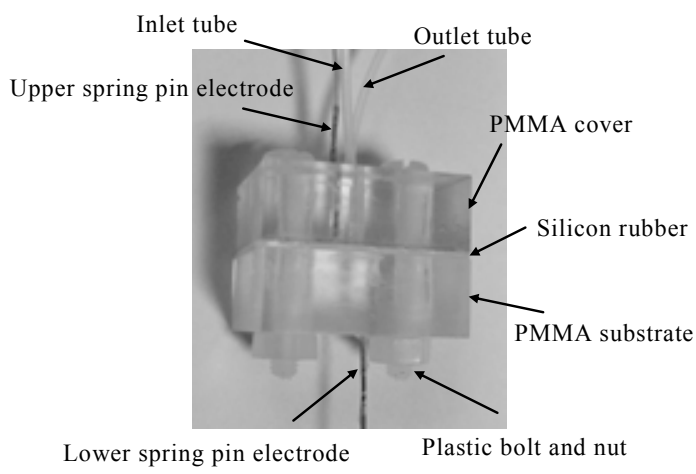

Fig. 5. Custom-made PMMA flow cell.

of a simple and inexpensive fabrication process over that described in ref. 15. To examine the performance of the custom-made flow cell, the vibration characteristics of the QCM chip are measured while equipped with the flow cell. Figures 6(b)-6(d) show the measured results after the QCM chip is assembled in the custom-made flow cell. The Q value dropped from 29000 to 23000 [as shown in Fig. 6(b)] in air, which indicates that the QCM chip suffered little mechanical stress from the sealing rubber or PMMA substrate. However, the degradation is unavoidable when the QCM chip is packaged; generally, it is thought that the $\mathrm{Q}$ value falls to a value below the half-value before it is assembled. ${ }^{(8,16)}$ Figures 6(c) and 6(d) show the vibration characteristics when flowing pure water and PBS solution through the QCM surface, respectively. The Q value fall to 1000 and 650 in air and PBS solution, respectively. The large difference 


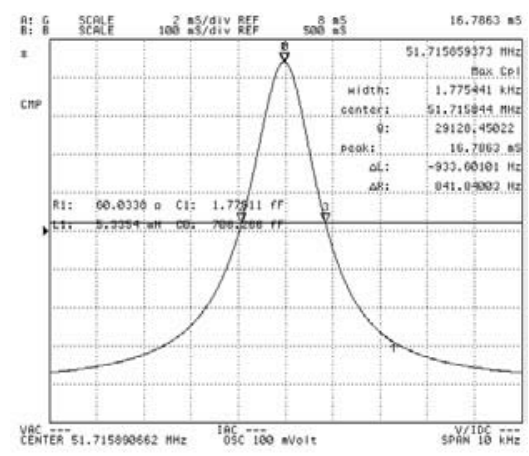

(a)

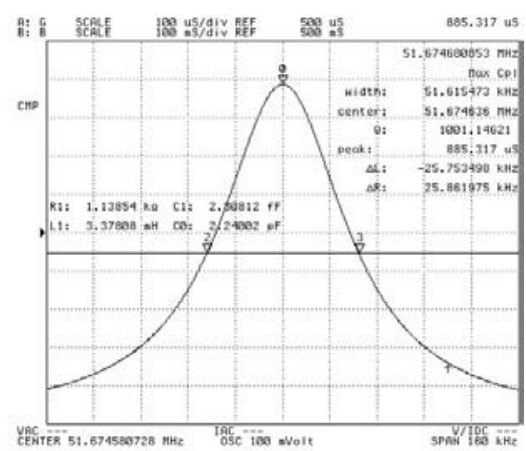

(c)

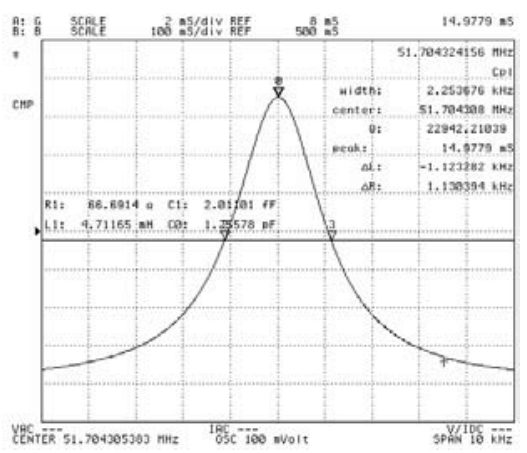

(b)

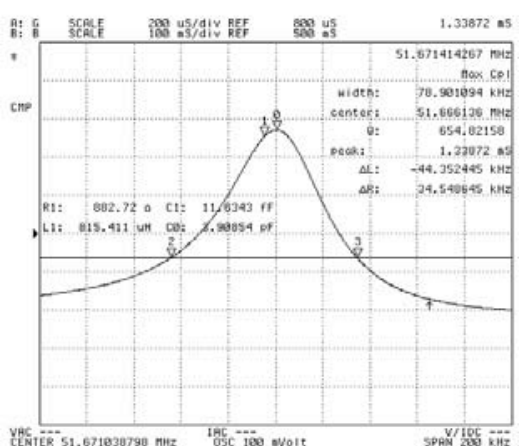

(d)

Fig. 6. Measured results using impedance analyzer 4294A: (a) using test fixture 16034G in air, (b) assembled in flow cell in air, (c) flowing in pure water, and (d) flowing in PBS solution.

between the $\mathrm{Q}$ value in air and that in liquid is due to the viscosity and density of the liquid. Theoretically, the maximum $\mathrm{Q}$ value is dependent on the resonator fundamental frequency, liquid viscosity and density. ${ }^{(15)}$ The measured data is in good agreement with that of the previously reported flow-cell-based high-frequency QCM sensor. ${ }^{(8,15)}$ These results indicate the success of the design and fabrication of the flow cell and demonstrate that the fabricated HFF QCM chip and flow injection system could be used as biosensors or chemical sensors. The advantages of our work over others are the miniaturization capability, simplicity, cost-effectiveness, fast and reliable installation, and reusability.

The above-mentioned results are all measured using an impedance analyzer, which suffers from high-frequency noise owing to the broadband scanning range as reported in ref. 8 and the long electrical connection. For reducing the frequency noise and actual applications, a high-quality, high-frequency oscillating circuit with a narrow bandwidth is desired, which would also make the sensing system more compact and portable. 


\section{Conclusions}

A flow-cell-based high-frequency QCM sensing system allowing for simple and efficient use as biosensors or chemical sensors is achieved. The designed and fabricated miniaturized high-frequency QCM chip at $52 \mathrm{MHz}$ with a size as small as $4 \times 4 \mathrm{~mm}^{2}$ is demonstrated to be suitable for installation into the proposed flow cell. By using an impedance analyzer, the fabricated $52 \mathrm{MHz}$ QCM chip equipped with the flow cell is evaluated to have high Q values of 23000 in air, 1000 in pure water, and 650 in PBS solution. These results demonstrate the success of the flow-cell-based high-frequency QCM sensor. The sensor not only has a high sensitivity, but also requires a small sample volume for measurement. The fabrication methods for both the high-frequency QCM chip and the flow cell are simple and cost-effective.

For practical applications, further development will be focused on studying a highquality oscillating circuit to reduce the frequency noise and on making the system more portable.

\section{Acknowledgements}

This work was financially supported by the Ph.D. Programs Foundation of the Ministry of Education of China (Grant No. 20110092120052), the Natural Science Foundation of Jiangsu Province (Grant No. BK2011607), and the Fundamental Research Funds for the Central Universities (China).

\section{References}

1 G. Z. Sauerbery: Z. Phys. 155 (1959) 206.

2 Z. Lin, C. M. Yip, I. S. Joseph and M. D. Ward: Anal. Chem. 65 (1993) 1546.

3 B. Zimmermann, R. Luchlum, P. Hauptmann, J. Rabe and S. Büttgenbach: Sens. Actuators, B 76 (2001) 47.

4 J. Rabe, S. Büttgenbach, J. Schröder and P. Hauptmann: IEEE Sens. J. 3 (2003) 361.

5 V. N. Hung, T. Abe, P. N. Minh and M. Esashi: Appl. Phys. Lett. 81 (2002) 5069.

6 T. Abe, V. N. Hung and M. Esashi: IEEE Trans. Ultrason. Ferroelectr. Freq. Control 53 (2006) 1234.

7 M. Michalzik, R. Wilke and S. Büttgenbach: Sens. Actuators, B 111-112 (2005) 410.

8 B. P. Sagmeister, I. M.Graz, R. Schwödiauer, H. Gruber and S. Bauer: Biosens. Bioelectron. 24 (2009) 2643.

9 E. Uttenthaler, M. Schräml, J. Mandel and S. Drost: Biosens. Bioelectron. 16 (2001) 735.

10 R. D. Williams, A. K. Upadhyayula and V. R. Bhethanabotla: Sens. Actuators 122 (2007) 635.

11 P. Kao, A. Patwardhan, D. Allara and S. Tadigadapa: Anal. Chem. 80 (2008) 5930.

12 P. Kao, S. Doerner, T. Schneider and D. Allara: J. Microelectromech. Syst. 18 (2009) 522.

13 T. Lederer, B. P. Stehrer, S. Bauer, B. Jakoby and W. Hiler: Sens. Actuators, A 172 (2011) 161.

14 J. Liang, X. Li, H. Li and T. Ueda: Proc. MNC2011, ed. H. Yamaguchi (JSAP, Kyoto, 2011) 27P-11-117 (CD-ROM).

15 P. Kao, D. Allara and S. Tadigadapa: Meas. Sci. Technol. 20 (2009) 124007.

16 H. Sota, H. Yoshimine, R. F. Whittier, M. Gotoh, Y. Shinohara, Y. Hasegawa and Y. Okahata: Anal. Chem. 74 (2002) 3592. 